\title{
Forms of collaboration matters: CSCL across the contexts
}

\author{
Sanna Järvelä ${ }^{1}$. Carolyn Rosé ${ }^{2}$ \\ Published online: 5 June 2021 \\ (c) International Society of the Learning Sciences, Inc. 2021
}

The field of CSCL has provided ample empirical evidence for principles underlying effective design and use of CSCL technologies, such as scripts and prompts, which provide ways of supporting cognitive and social interactions for better collaboration. Nevertheless, though this empirical foundation suggests that collaborative learning is a powerful learning method, even demonstrating its potential in remote teaching and learning, we have somehow not seen this in evidence globally in this recent pandemic. This situation prompts us to reflect once again on what we know and what we still need to know. And furthermore, we prepare to look back on our experiences during this time to see how our lives online have transformed our world, shaping new networks for research and life online. In that vein, the research papers in this issue discuss forms of collaboration, their value, contribution and analysis across the different contexts, with a common thread of networks, as analytic lenses, instructional representations, and social constructs. We include four full articles, the first two with a backward glance, analyzing social networks first in recent history and then in the distant past, followed by two articles related to supportive technologies for fostering connection and learning within social configurations. We conclude with a Squib, again prompted by the recent pandemic, with a forward looking vision that calls for a more intensive theoretical integration of cognitive and social perspectives on learning as we consider what our field has to say about fostering connection in learning environments going forward.

Collaborations are essential in research, especially in answering increasingly complex questions that require integrating knowledge from different disciplines and that engage multiple stakeholders. Integration of multiple perspectives is a value at the heart of this field, and a running theme through the papers of this June edition. Collaborations occur across scientific society, teams, career levels, disciplines, and various technologies to support it. In particular, the recent pandemic has reminded us of the immense value of faceto-face collaborative interactions as we observe the effects of living without the ability to work face-to-face, meet in conferences, or even meet over coffee.

Carolyn Rosé

cp3a@andrew.cmu.edu

Sanna Järvelä

sanna.jarvela@oulu.fi

1 University of Oulu, Oulu, Finland

2 Carnegie Mellon University Language Technologies Institute and HCI Institute, Pittsburgh, PA, USA 
Julia Eberle, Karsten Stegmann, Alain Barrat, Frank Fischer and Kristine Lund study about scientific collaborations across career levels and disciplines. This message couldn't be timelier for the purpose of reminding us of the importance of social networking and collaborations in scientific communities and the progress of scientific knowledge in general. During the past year our face-to-face scientific collaboration has been minimal, while online collaboration and distant interaction maximized. CSCL researchers are also increasingly collaborating in multidisciplinary teams for answering complex interdisciplinary research questions and integrating new technological and analytical solutions to understand and support collaborative learning. But how are these collaborations instigated? If we can learn more about how these collaborations are sparked, we might be able to increase their occurrence. What is exciting in the Eberle et al. study is that they investigated "us" - data were collected at two CSCL scientific events including 5736 relations between 287 researchers. They investigated how researchers selected future collaboration partners, looking specifically at the role of career level, disciplinary background, and selection patterns. Specifically, they studied how participants at face-to-face meetings in the interdisciplinary field of technology-enhanced learning select new collaboration partners and what may be influential factors in this process.

Face-to-face contact was measured using RFID devices and questionnaire data were collected. Additionally, a group awareness intervention was experimentally varied. Data was analysed using RSiena and meta-analyses. The results showed that transitivity, reciprocity and contact duration are relevant for the identification of new potential collaboration partners. PhD students were less often chosen as new potential collaboration partners and researchers with a background in Information Technology selected fewer new potential collaboration partners. However, group awareness support balanced this disciplinary difference. The Eberle et al. study clearly sheds light on the way research collaborations evolve, but also how it can be instigated and supported in informal settings.

While the first paper utilized social network analysis as a lens for studying interactions within our own community, the second paper uses network representations as an instructional representation for understanding social networks of the distant past in history instruction. Specifically, Kalani Craig, Joshua Danish, Megan Humburg, Cindy HmeloSilver, Maksymilian Szostalo and Ann McCranie present Net.Create: Network Visualization to Support Collaborative Historical Knowledge Building. While much of the published research on formal instruction focuses on STEM fields, this paper fills a needed gap in terms of its investigation of learning in history. While misconceptions exist that history is really about memorization of names and dates, the article underscores the value of delving deeper into the multiple perspectives on why events occur and how they are interconnected. This perspective harkens back to past work on delving into different perspectives on responsibility for politically charged historical events (Pollack \& Kolikant, 2012).

Craig et al. present the Net.Create platform, which affords the opportunity for students to co-construct a network representation of pieces of history, in ways that enable them to reason separately and together not only about what happened, but why, and how that reveals important connections between people and between events over time. Using Activity Theory as a lens, they investigate how students work together using Net.Create to develop their understanding of the distant past, in the time of Claudius, Agrippina, and Nero. Their in depth process analysis enables us to see how collaborative reasoning can evolve over two days as students explore their growing understanding of this historical period through their interactions with the text through Net.Create.

Just as we as researchers use network representations to model large scale social interaction, students in the Craig et al. study push beyond a simple recounting of links, weights, 
and network structures to layer their own interpretation over the structures that serve as scaffolding for their joint reasoning. This portal into the development of historical reasoning provides inspiration regarding the potential of network representations to serve as boundary objects for researchers to reason together over multiple interpretations about what might underlie the social processes that played out over time. Perhaps we as a society could take inspiration from this work as we consider what we have learned about our own history from the Eberly et al. study.

While group awareness (GA) tools have been successful in CSCL facilitating learning processes (e.g. Janssen \& Bodemer, 2013), combining a variety of awareness information through combinations of GA tools has been under explored. Lisa Ollasch, Sven Heimbuch and Daniel Bodemer argue that to assist learning and writing in social media, combining a variety of awareness information may foster learning processes for challenges that are difficult to address by one type of GA alone. To reveal both positive and negative interaction effects of providing different types of GA information, Ollasch et al. conducted an experimental study with 158 participants. Learners were provided with a wiki learning environment and, except for the control condition, different types of GA tools involving cognitive and/or behavioral GA information. GA tool effects were analyzed at wiki selection, discussion, and article levels, and eye-tracking was used for investigating the attentional effect of the GA visualizations. Their results show that providing users with cognitive and behavioral GA information can have positive effects on cognitive as well as behavioral learning processes and outcomes in social media. These results contribute to practical solutions for supporting wikis and other CSCL environments. However, the results also highlight that cognitive and behavioral GA combinations do not necessarily have a positive effect on emotional outcomes of learners, such as well-being. These results point to the necessity of considering emotional processes in learning contexts. Based on their findings, the authors call for more research on behavioral, cognitive, and emotional processes and outcomes on different levels for understanding and using the full potential of GA support in social media contexts.

While the role of support in CSCL research in the form of scaffolds, prompts, tutors and tools is one of the primary research agendas of the field, the role of the teacher in supporting and guiding collaborative learning has gotten less attention. Tarja Pietarinen, Tuike Iiskala and Marja Vauras studied the real-time focus and distribution of teachers' guidance and support of different student groups during in-person computer-supported collaborative inquiry learning in science classrooms. The virtual learning environment, Virtual Baltic Sea Explorer (ViBSE), was used to support students' collaborative learning in science, specifically studying environmental changes in the Baltic Sea. Observing collaborative groups and their temporal progress during group tasks is particularly important since recent studies have pointed out problems in the process of collaboration in terms of social interactions and group dynamics, motivation and groups' regulatory processes (e.g., Bakhtiar \& Hadwin, 2020). For example, while studies implementing multimodal data channels and collaborative analytics (e.g., Martinez-Maldonado et al., 2021) have considerably advanced the field through analysis of collaborative learning traces and patterns of activities, the findings so far lack the power required to explain the qualities of teacher student interactions and their contextual conditions.

The Pietarinen et al. study focuses on teacher activity, teachers' self- perception of their own guidance and affect, and student-reported affect. Four high school science teachers and the students they taught in each of their four classes participated in the study. Data was collected by videotaping the lessons and self-report instruments focusing on teacher and student affect. An in-depth analysis focused on the intertwined connections of teacher guidance, 
support and learning outcomes. This analysis revealed differences between teacher guidance and support depending on teacher perceptions and group outcomes. Groups' prior science competence was not found to have an effect on teacher guidance and support. Teacher affect was compounded by student affect, suggesting that reciprocal perceptions are necessary for understanding the teachers' role in collaborative learning. This study highlights the value of understanding nuances of collaboration and understanding the teachers' role in facilitating CSCL.

Jaana Isohätälä, Piia Näykki, Sanna Järvelä, Michael Baker and Kristine Lund squib in this issue call for social sensitivity in CSCL research. The authors argue that while novel technologies are already changing the way people learn together, they don't yet foster engagement in constructively critical, respectful, and cohesive collaborations. They urge CSCL researchers to explore the concept of social sensitivity in CSCL. Drawing on the core concepts of CSCL (collaboration, co-construction, knowledge building and social interaction), they broadly define social sensitivity as people's individual and collective ability to collaborate constructively, respectfully, and cohesively. They also introduce social sensitivity as an overarching term to view the complexity of CSCL research and the range of empirical evidence that must be acknowledged when developing and applying technologies for collaborative learning. While they acknowledge that CSCL is a multidisciplinary framework housing a multitude of approaches (Stahl, 2013), their claim is that the CSCL community is too willing to simply accept the coexistence of parallel research paradigms and concepts, with too little debate about the conflicts or synergies between them.

The squib continues the discussion in our journal started by Uttamchandani et al. (2020) who called upon researchers to focus on equity in CSCL practices in order to promote educational change. Isohätälä et al. call for actions in CSCL research that ultimately contribute to more democratic and equitable collaborations. They bring forward a manifesto of social sensitivity: increasing interdisciplinary efforts to enhance constructively critical, respectful, and cohesive collaborations in technology-supported environments. By the manifesto they suggest CSCL researchers take concrete actions in the pursuit of a more coherent vision of social sensitivity in CSCL drawing from three perspectives. First, they call for crossing theoretical silos by increasing efforts in clarifying differences, overlaps and relations between concepts. Second, that call for accelerating interdisciplinary, multimodal empirical research by forming new interdisciplinary partnerships where the shared goal is to provide evidence about how emerging technologies influence social sensitivity in CSCL. Third, they call for transferring evidence to practice and policy using social sensitivity as a theme for interaction with educational technology developers, learning designers, teachers, and students.

We hope that this issue will encourage researchers to keep on studying the interactions and forms of collaboration that are at the core of explanations of successful collaborative learning, but also, as shown in the research papers of this issue, the key to widening representation of collaborative learning contexts with the aim of making the CSCL field more powerful. We challenge the field to continue the discussion about social sensitivity and re-new the field by opening new avenues of thinking for further debate.

\section{References}

Bakhtiar, A., \& Hadwin, A. F. (2020). Dynamic interplay between modes of regulation during motivationally challenging episodes in collaboration. Frontline Learning Research, 8(2), 1-34. https://doi.org/10. 14786/flr.v8i2.561 
Janssen, J., \& Bodemer, D. (2013). Coordinated computer-supported collaborative learning: Awareness and awareness tools. Educational Psychologist, 48(1), 40-55. https://doi.org/10.1080/00461520.2012. 749153

Martinez-Maldonado, R., Gašević, D., Echeverria, V., Fernandez Nieto, G., Swiecki, Z., \& Buckingham Shum, S. (2021). What do you mean by collaboration analytics? A conceptual model. Journal of Learning Analytics, 8(1), 126-153.

Pollack, S., \& Kolikant, Y. B. D. (2012). Collaboration amidst disagreement and moral judgment: The dynamics of Jewish and Arab students' collaborative inquiry of their joint past. International Journal of Computer-Supported Collaborative Learning, 7(1), 109-128. https://www.learntechlib.org/p/ $110726 /$.

Stahl, G. (2013). Theories of cognition in collaborative learning. In C. E. Hmelo-Silver, C. A. Chinn, C. Chan, \& A. M. O'Donnell (Eds.), The International Handbook of Collaborative Learning (pp. 74-90). Routledge.

Uttamchandani, S., Bhimdiwala, A., \& Hmelo-Silver, C. E. (2020). Finding a place for equity in CSCL: ambitious learning practices as a lever for sustained educational change. International Journal of Computer-Supported Collaborative Learning, 373-382. https://doi.org/10.1007/s11412-020-09325-3.

Publisher's note Springer Nature remains neutral with regard to jurisdictional claims in published maps and institutional affiliations. 